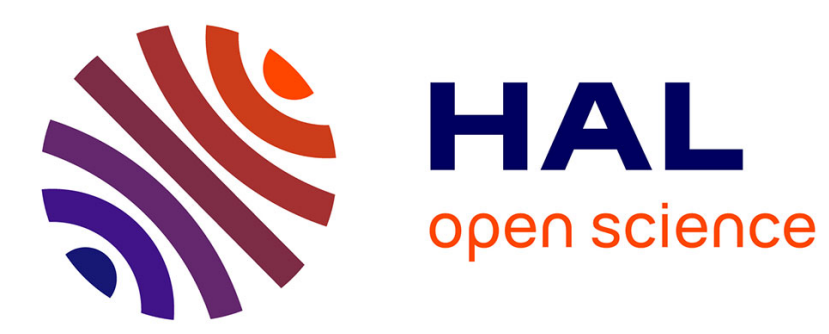

\title{
Drag Reduction of the Plane Poiseuille Flow by Partitioned Visual Servo Control \\ Xuan-Quy Dao, Christophe Collewet
}

\section{To cite this version:}

Xuan-Quy Dao, Christophe Collewet. Drag Reduction of the Plane Poiseuille Flow by Partitioned Visual Servo Control. American control conference, Jun 2012, Montréal, Canada. pp.4084-4089. hal-00726528

\section{HAL Id: hal-00726528 \\ https://hal.inria.fr/hal-00726528}

Submitted on 30 Aug 2012

HAL is a multi-disciplinary open access archive for the deposit and dissemination of scientific research documents, whether they are published or not. The documents may come from teaching and research institutions in France or abroad, or from public or private research centers.
L'archive ouverte pluridisciplinaire HAL, est destinée au dépôt et à la diffusion de documents scientifiques de niveau recherche, publiés ou non, émanant des établissements d'enseignement et de recherche français ou étrangers, des laboratoires publics ou privés. 


\title{
Drag Reduction of the Plane Poiseuille Flow by Partitioned Visual Servo Control
}

\author{
Xuan-Quy Dao and Christophe Collewet
}

\begin{abstract}
We present in this paper a way to minimize the drag of the 2D plane Poiseuille flow. To do that, as in our previous work [1], we use a vision-based approach to estimate the state of the flow. Since visual measurements are used, we propose to exploit visual servoing techniques to derive an efficient control law. Therefore, contrary to the literature concerning drag reduction, we also simultaneously minimize the kinetic energy density of the flow. That is of great importance since the controlled flow may become turbulent when this kinetic energy density is growing. To cope with this problem we propose to design a control law based on partitioned visual servo control. This approach has been first proposed in the robotics community by [2]. Simulations results validate our control scheme. We also compare our approaches with the most relevant ones.
\end{abstract}

\section{INTRODUCTION}

The goal of this paper is to show the benefit of using advanced visual servoing techniques [3] for fluid flow control. By controlling a flow we mean either to change its state to another state or to maintain its current state whatever external disturbances. This can be of great environmental and economical interests for industrial applications. For instance, fuel consumption of aircrafts can be highly reduced by decreasing the drag while enhancing the lift (up to $20 \%$, see [4]).

Flow control can be achieved in two different ways: passive or active control. Passive control provides a permanent action on a system. Most often it consists in optimizing shapes or in choosing suitable surfacing. Conversely, in active control an external energy is required to act on the system like, for example, techniques based on blowing and suction [5]. This approach can be seen as an optimal problem where one has to apply an optimal control law based on a certain cost (minimization of the drag, minimization of the actuators power, etc.) [6]. However, very often, only openloop control or even most often forcing is used [7]. Indeed, using a closed-loop control law requires sensors that have to be at the same time non-intrusive, accurate and adapted to the time and space scale of the phenomenon that we have to control. Unfortunately, such sensors are hardly available in the context of real control applications. The most commonly used measurement, obtained from Micro Electro Mechanical Systems (MEMS), is the shear stress at a limited set of measurement points [5]. Thereafter, an observer is required to estimate the state of the flow that will be used in the

X-Q. Dao and C. Collewet are with INRIA Rennes Bretagne Atlantique, Fluminance team, Rennes, France. E-mail: firstname.namedinria.fr. control law. Indeed, the shear stress based LQG regulator is the standard effective approach for flow control [5], [6], [8].

In our previous paper [1] this estimation is obtained from visual measurements. More precisely, the optical flow [9] is computed and used to build an observer-free closed-loop control law as detailed later. Our approach has been revealed to be much more robust than the classical approaches [5], [6], [8]; it is no more concerned with the well known problem of initialization issue when an observer is used. It has also been shown to be highly robust to measurements noise since a large number of measurements is available. To compare our approach with the classical one we also used the LQG control law to control the flow. In this paper, since visual measurements have been used, we aim to fully exploit the capabilities of visual servoing techniques by designing a more efficient control law.

Visual servoing or visual servo control aims to control the motions of a robot by using data provided by a vision sensor [10]. It is now a well established technique in the robotics community. More precisely, to achieve a visual servoing task, a set of visual features $\mathbf{s}(t)$ is selected from the image of the scene being observed. A control law is then designed so that this set of visual features $\mathbf{s}(t)$ reaches a desired value $\mathbf{s}^{*}$ corresponding to a desired state of the system. The dynamic of the error vector $\mathbf{e}(t)=\mathbf{s}(t)-\mathbf{s}^{*}$ is then given by

$$
\dot{\mathbf{e}}(t)=\frac{\partial \mathbf{e}(t)}{\partial t}+\mathbf{L}_{\mathbf{e}}(t) \mathbf{u}(t),
$$

where $\mathbf{u}(t)$ is the system control inputs, $\mathbf{L}_{\mathbf{e}}(t)$ is the so-called interaction matrix [11] or the image jacobian that links the time variation of the visual features to the variation of the control signal acting on the system and $\partial \mathbf{e}(t) / \partial t$ expresses the variation of the error vector due to the free motion of the visual features. The control principle is of course to regulate the error vector e to zero. The control law is built from (1) using the knowledge or an approximation of the interaction matrix and an approximation of the free motion of the visual features. Visual servoing has shown impressive results in numerous complex contexts such as underwater, medical and aerial (helicopters, blimps) robotic as shown in [12].

The final goal of this paper is to reduce the skin friction drag. As already mentioned at the top of this introduction, reducing the drag is an important challenge. Drag reduction in turbulent boundary layers has been investigated in the recent years. An adjoint-based suboptimal control approach has been proposed in [13], a drag reduction of approximately $17 \%$ has been obtained. However this way to proceed leads 
to a very high computation cost, not compatible within a real time control scheme. Moreover, it is not possible to implement this approach in practice, see [14] for more details. More viable approaches has been proposed in [5], [15]-[17]. In [5], a simple proportional controller was used to suppress the wall shear stress leading consequently to a drag reduction, while in [15]-[17] a LQR approach has been used in which the cost function to minimize includes the drag. However, all these works does not account the kinetic energy density of the flow. This is yet an important issue since it is well known that if this density of energy is growing, transition of the controlled flow to turbulence is possible. That is why lots of papers focused on this problem (see e.g. [18], [19]).

The main contribution of this paper is to show that, by fully exploiting the controlled degrees of freedom, a control law can be derived to simultaneously reduce the drag and the kinetic energy density of the flow. We will validate this approach on the 2D plane Poiseuille flow (see a description of this flow in section II). Note that an interesting approach has been proposed in [20], in this work both a drag reduction and a reduction of the kinetic energy density have been obtained. However, tangential actuation has been used. This way to acts has been revealed to lead to lower controllability than wall normal actuation (see [21]). This is why, the more classical wall normal actuation is preferred in this paper as for example in [5], [6], [8], [15]-[17].

This paper is organized as follows: in Section II we first present some basics of the $2 \mathrm{D}$ plane Poiseuille controlled flow and the modeling model of some physical quantities that will be involved in the control law. The control scheme is detailed in Section III while in Section IV the simulation results are presented, we compare our approach with the most relevant ones.

\section{MODELING ISSUES FOR CONTROL DESIGN}

In this section we first present some basics of the plane Poiseuille flow, then we introduce the control principle for this flow and present a reduced linearized model around the steady state. The modeling of skin friction drag and the kinetic energy density are next presented.

\section{A. Basics of the plane Poiseuille flow}

Poiseuille flow is a flow in an infinite length channel due to a pressure gradient. Fig. 1 illustrates the steady state velocities profile of the plane Poiseuille flow in a streamwise period of the infinite length channel according to the conceptual model proposed in [5]. The $x$ axis corresponds to the streamwise direction and the $y$ axis to the wall normal direction. The non dimensionalized Navier-Stokes equation of this flow is given by

$$
\left\{\begin{array}{l}
\frac{\partial \mathbf{V}}{\partial t}+(\boldsymbol{\nabla} \mathbf{V}) \mathbf{V}=-\boldsymbol{\nabla} P+\frac{1}{R_{e}} \nabla^{2} \mathbf{V} \\
\boldsymbol{\nabla} \cdot \mathbf{V}=0 \\
\mathbf{V}(x, y= \pm 1, t)=\mathbf{0}
\end{array}\right.
$$

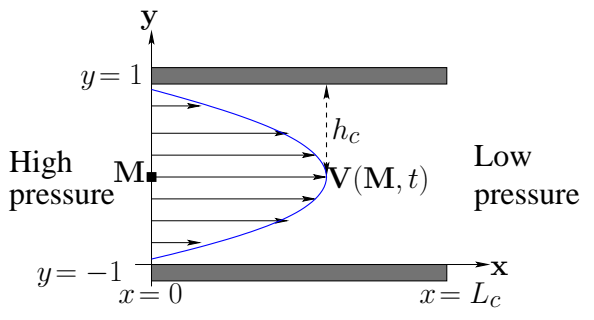

Fig. 1. Steady state velocities profile of the 2D plane Poiseuille flow: $h_{c}$ is the channel half height, $L_{c}$ is the flow streamwise period length and $\mathbf{M}$ is a point in the flow.

where $P$ is the pressure; $\mathbf{V}$ is the flow velocity; $\mathbf{V}(x, y= \pm 1, t)=\mathbf{0}$ represents the no slip boundary condition; $R_{e}$ is a dimensionless number called the Reynolds number; $\boldsymbol{\nabla}=\left(\frac{\partial}{\partial x}, \frac{\partial}{\partial y}, \frac{\partial}{\partial z}\right)$ represents the gradient operator and $\nabla^{2}$ the Laplacian.

Since the Poiseuille flow is simple, the analytical solution $\left(V_{b x}, V_{b y}, P_{b}\right)$ of (2) in the steady state case, i.e. $\frac{d \mathbf{V}}{d t}=\frac{\partial \mathbf{V}}{\partial t}+\mathbf{V} \cdot \nabla \mathbf{V}=\mathbf{0}$, can be found:

$$
\left(V_{b x}, V_{b y}, P_{b}\right)=\left(1-y^{2}, 0,-\frac{2}{R_{e}} x\right) .
$$

This solution is illustrated in Fig. 1.

\section{B. Boundary control of the Poiseuille flow}

The typical way to control the 2D plane flow Poiseuille is boundary control.

It consists in modifying the boundary conditions of the system (2) either only on the lower boundary $y=-1$ [5], or on both the upper $y=1$ and lower $y=-1$ boundaries [8]. Boundary control on the upper and the lower channels can be theoretically represented by functions $\chi_{u}$ and $\chi_{l}$ that allows mass conservation in the controlled system as pictured in Fig. 2.

Practically, fluidic devices (mean to inject and to suck fluid) like synthetic jet actuators can be used. Note those actuators are only one possible type of actuators for flow control since there are numerous other actuator control mechanisms such as plasma or other type which involves moving the domain boundary as described in [22].

\section{Reduced linearized model of Poiseuille flow}

The modeling, required to derive the control law, consists first of all in linearizing the Navier-Stokes equation around the steady state solution (3). Then the continuous linearized model of this equation is first represented in the Fourier domain at a specific wavenumber $\alpha_{n}=2 \pi / L_{c}$ containing the instability [23]: $\mathbf{V}_{p}(x, y, t)=\mathbf{V}_{p}^{n}(y, t) e^{j \alpha_{n}}$. Then the spectral representation $\mathbf{V}_{p}^{n}(y, t)$ is discretised in the $y$ direction by using a number of $M$ Gauss-Lobatto collocation points $y_{k}=\cos ((k-1) \pi /(M-1)), 1 \leq k \leq M$ leading to the following reduced linearized model (see [8] for all the details):

$$
\left\{\begin{array}{l}
\dot{\mathbf{x}}^{n}(t)=\mathbf{A}^{n} \mathbf{x}^{n}(t)+\mathbf{B}^{n} \mathbf{u}(t) \\
\mathbf{x}^{n}(0)=\mathbf{x}_{0}^{n}
\end{array}\right.
$$

where $\mathbf{x}^{n}(t)$ is the state vector of dimension $M, \mathbf{A}^{n}$ is the state matrix which dimensions are $M \times M, \mathbf{u}(t)=$ 


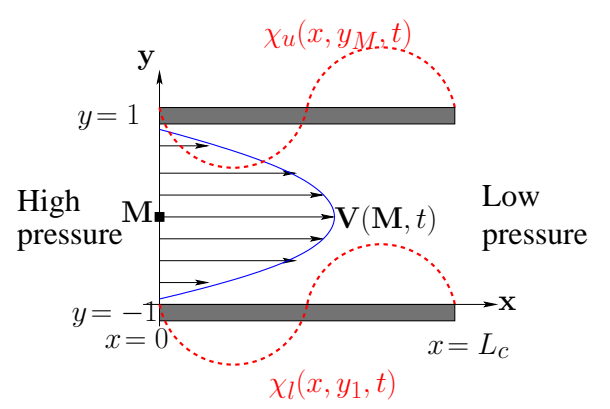

Fig. 2. Boundaries control of the plane Poiseuille flow.

$\left(u_{u}(t), u_{l}(t)\right)$ is the system input (i.e. blowing and suction actions at the channel boundaries), $\mathbf{B}^{n}$ is the input matrix which dimensions are $M \times 2$. Recall that in our case (see [1]) the state vector is directly obtained from the computation of the optical flow through a visualization system as shown in Fig. 3. More precisely, optical flow is the apparent velocity vector field representing the motion of photometric pattern (pixels luminance) in successive image sequences. Optical flow techniques can be used to estimate instantaneous velocities of a fluid flow from any image sequences as detailed in [9].

In the case of Reynolds number $R e=10000$ and wavenumber $\alpha_{n}=1$, the reduced linearized model of Poiseuille flow (4) is unstable as shown in [23]. Therefore, as in [5], [8], [6] we focus on the stabilization of the flow, i.e. maintaining the flow in the steady state, whatever the external perturbations. In this case the flow is initially in the steady state but in an unstable equilibrium, i.e. a small velocity pertubation value $\mathbf{V}_{p}(x, y, t)$ destabilizes the non-controlled fluid flow. This instability can be easy seen through the poles of the matrix $\mathbf{A}^{n}$, a pole has a real part positive $(\lambda=0.00373967 \pm j 0.23752649)$. We will consider this case in the remainder of this paper. Note also that we will omit now the upperscript $n$ involved in (4) for the sake of clarity.

\section{Modeling of the skin friction drag}

The skin friction drag $d(t)$ due to the perturbation is given by (see e.g. [20])

$$
d(t)=-\frac{\partial V_{p x}(x,+1, t)}{\partial y}+\frac{\partial V_{p x}(x,-1, t)}{\partial y}
$$

where $V_{p x}(x,+1, t)$ and $V_{p x}(x,-1, t)$ are the streamwise components of the perturbation velocity $\mathbf{V}_{p}(x, y, t)$ at the walls. Note that $\partial \mathbf{V}_{p x}(x,-1, z, t) / \partial y$ and $\partial \mathbf{V}_{p x}(x,+1, z, t) / \partial y$ are the components of the wall shear stress $\mathbf{s}(t)$ due to the perturbation:

$$
\mathbf{s}(t)=\left[\begin{array}{l}
\frac{\partial V_{p x}(x,+1, z, t)}{\partial y} \\
\frac{\partial V_{p x}(x,-1, z, t)}{\partial y}
\end{array}\right] .
$$

According to modeling process recalled in section II-C, it can be shown that $\mathbf{s}(t)$ writes simply as a linear function of the state through a matrix $\mathbf{W}[8]$

$$
\mathbf{s}(t)=\mathbf{W} \mathbf{x}(t)
$$

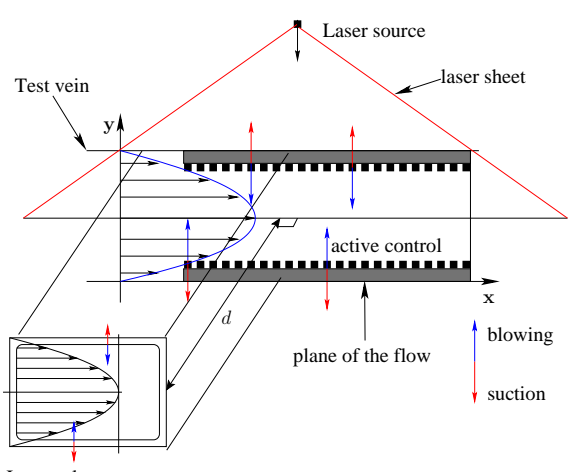

Image plane

Fig. 3. Visualization of the flow using a laser sheet which role is to enlighten the particles seeded in the fluid.

leading consequently from (5) to

$$
d(t)=\mathbf{D}^{\top} \mathbf{x}(t)
$$

with $\mathbf{D}^{\top}=\left[\begin{array}{ll}-1 & +1\end{array}\right] \mathbf{W}$ where $\mathbf{D}^{\top}$ denotes the conjugate transpose of $\mathbf{D}$.

\section{E. Modeling of the kinetic energy density}

The kinetic energy density of flow perturbation is given by

$$
\varepsilon(t)=\frac{1}{V_{0}} \int_{V_{0}} \frac{\left\|\mathbf{V}_{P}(x, y, t)\right\|^{2}}{2} d V_{0}
$$

where $V_{0}$ is the volume of a period of the domain under consideration. It is possible to obtain a weighting matrix $\mathbf{Q}$ exactly as in [19] such that the kinetic energy density reduces to

$$
\varepsilon(t)=\mathbf{x}(t)^{\top} \mathbf{Q} \mathbf{x}(t)
$$

All these physical quantities having been defined, the control law can now be derived.

\section{Control of the 2D Plane Poiseuille flow}

As mentioned in the introduction of this paper, our goal is to simultaneously minimize the drag and the kinetic energy density since the flow is in unstable state (see section IIC). To achieve this goal we propose to use the so-called partionned visual servo control [2]. It is a well known approach in the robotics community used to decouple the rotational motions from the translational ones. To do that, we first have to compute the interaction matrix $\mathbf{L}_{d}$ related to the drag.

\section{A. Computation of the interaction matrix related to the drag}

This computation is straightforward. Indeed, according to definition of the interaction matrix [11], we have to express the total time variation of the drag

$$
\dot{d}(t)=\mathbf{L}_{d} \mathbf{u}(t)+\frac{\partial d(t)}{\partial t} .
$$

$\mathbf{L}_{d}$ encodes the variation of the drag due to the actions, while $\partial d(t) / \partial t$ expresses the variation of the drag due to the flow itself. 
This computation can be done by derivating (8) with respect to the time and next using (4). We obtain

$$
\left\{\begin{array}{c}
\mathbf{L}_{d}=\mathbf{D}^{\top} \mathbf{B} \\
\frac{\partial d(t)}{\partial t}=\mathbf{D}^{\top} \mathbf{A x}(t)
\end{array}\right.
$$

Let us introduce the components of the interaction matrix related to the components of the input signal $\mathbf{u}(t)$ (see (4)).

$$
\mathbf{L}_{d}=\left(L_{d u}, L_{d l}\right) \text {. }
$$

This expression will be useful in the next section to introduce the partitioned visual servo control.

\section{B. Design of the control law}

Partitioned visual servo control relies on the partition of the interaction matrix. From (13), the time variation of the drag (11) becomes

$$
\dot{d}(t)=L_{d u} u_{u}(t)+L_{d l} u_{l}(t)+\frac{\partial d(t)}{\partial t} .
$$

Since a decreasing of the drag is desired, we choose a particular behavior for the drag. An exponential decrease is imposed

$$
\dot{d}(t)=-\lambda d(t)
$$

where $\lambda$ is a positive gain able to tune the decrease rate. Thereafter, from (14) and (15), we have

$$
-\lambda d(t)=L_{d u} u_{u}(t)+L_{d l} u_{l}(t)+\frac{\partial d(t)}{\partial t} .
$$

Any of the two components of $\mathbf{u}(t)$ can be used to reduce the drag, we arbitrary choose $u_{l}(t)$. We thus have

$$
u_{l}(t)=-\frac{1}{L_{d l}}\left(\lambda d(t)+L_{d u} u_{u}(t)+\frac{\partial d(t)}{\partial t}\right) .
$$

Since the lower boundary control law $u_{l}(t)$ is known if the upper boundary control law $u_{u}(t)$ is known, the next step is to express $u_{u}(t)$.

In order to minimize the kinetic energy density (10) and the energy consumption of actuators, a LQR control scheme over an infinite time horizon is used by considering the following cost function

$$
J=\int_{0}^{\infty}\left(\mathbf{x}^{\top}(t) \mathbf{Q} \mathbf{x}(t)+r u_{u}^{2}(t)\right) \mathrm{d} t
$$

This can be done by expressing the time variation of the state vector with respect to the control signal $u_{u}$. To do that, we first rewrite (17) from (12) to exhibit the state vector

$$
u_{l}(t)=-\frac{1}{L_{d l}}\left(\left(\lambda \mathbf{D}^{\top}+\mathbf{D}^{\top} \mathbf{A}\right) \mathbf{x}(t)+L_{d u} u_{u}(t)\right)
$$

that we have to plug in (4) leading to

$$
\dot{\mathbf{x}}(t)=\mathbf{A}_{1} \mathbf{x}(t)+\mathbf{B}_{1} u_{u}(t)
$$

where we have introduced the following matrices $\mathbf{A}_{1}=$ $\mathbf{A}-\frac{B_{l}}{L_{d l}}\left(\lambda \mathbf{D}^{\top}+\mathbf{D}^{\top} \mathbf{A}\right)$ and $\mathbf{B}_{1}=\mathbf{B}_{u}-\frac{L_{d u}}{L_{d l}} \mathbf{B}_{l}$ with $\mathbf{B}=\left(\mathbf{B}_{u}, \mathbf{B}_{l}\right)$. Note that $L_{d l}$ is always non null.

Thereafter, it becomes straightforward to compute the LQR gain $\mathbf{k}$ involved in the optimal control $u_{u}=-\mathbf{k}^{\top} \mathbf{x}(t)$ by considering (20) and solving the algebraic Ricatti equation.

Note that here, contrary to the works involved in flow control, we have fully exploited the controlled degrees of freedom.

The next section is dedicated to the study of the behavior of the closed loop system when measurement noise is considered.

\section{Behavior of the closed loop system in presence of mea- surement noise}

Of course, when measurement noise occurs, the main problem is the stability analysis of (20). In that case, the upper control signal becomes

$$
\hat{u}_{u}=-\mathbf{k}^{\top} \hat{\mathbf{x}}(t) .
$$

However, it has been shown in [1] that in our vision-based approach the state estimation writes as

$$
\hat{\mathbf{x}}(t)=\mathbf{x}(t)+\frac{1}{N_{x}} \mathbf{e}_{x}(t)
$$

where $N_{x}$ is the number of pixels of the camera in the streamwise direction of the flow and $\mathbf{e}_{x}(t)$ is related to the measurements noise (see [1] for more details). It is clear from (22), that the larger the value of $N_{x}$ the closer $\hat{\mathbf{x}}(t)$ is from $\mathbf{x}(t)$. Consequently, from (21), (20) becomes

$$
\dot{\mathbf{x}}(t)=\left(\mathbf{A}_{1}-\mathbf{B}_{1} \mathbf{k}^{\top}\right) \mathbf{x}(t)-\mathbf{B}_{1} \mathbf{k} \boldsymbol{\delta}(t)
$$

where the estimation error $\boldsymbol{\delta}(t)=\hat{\mathbf{x}}(t)-\mathbf{x}(t)$ has been introduced. When $N_{x}$ is large enough (it is always the case in practice), the estimation error tends toward 0 , and, consequently the closed loop system (23) writes as

$$
\dot{\mathbf{x}}(t)=\left(\mathbf{A}_{1}-\mathbf{B}_{1} \mathbf{k}^{\top}\right) \mathbf{x}(t)
$$

which is stable.

The other issue concerns the drag. Indeed, we have to verify that it is still a decreasing function. To do that, we exam (14) by considering $\hat{u}_{u}, \hat{u}_{l}$ and $\partial \hat{d}(t) / \partial t$. From (19), we have

$$
\hat{u}_{l}(t)=-\frac{1}{L_{d l}}\left(\left(\lambda \mathbf{D}^{\top}+\mathbf{D}^{\top} \mathbf{A}\right) \hat{\mathbf{x}}(t)+L_{d u} \hat{u}_{u}(t)\right)
$$

that writes simply in function of $\mathbf{x}(t)$ by considering (21) and (22)

$\hat{u}_{l}(t)=-\frac{1}{L_{d l}}\left(\lambda \mathbf{D}^{\top}+\mathbf{D}^{\top} \mathbf{A}-L_{d u} \mathbf{k}^{\top}\right)\left(\mathbf{x}(t)+\frac{1}{N_{x}} \mathbf{e}_{x}(t)\right)$.

The last step is the computation of $\partial \hat{d}(t) / \partial t$, it can be easy done from (12) and (22). All computations done, (14) becomes

$$
\dot{d}(t)=-\lambda d(t)-\frac{\lambda \mathbf{D}^{\top}+\mathbf{D}^{\top} \mathbf{A}}{N_{x}} \mathbf{e}_{x}(t) .
$$

Here again, since $N_{x}$ is a large value, we can consider than the drag follows the desired behavior $\dot{d}(t)=-\lambda d(t)$.

These results will be validated in the next section. 

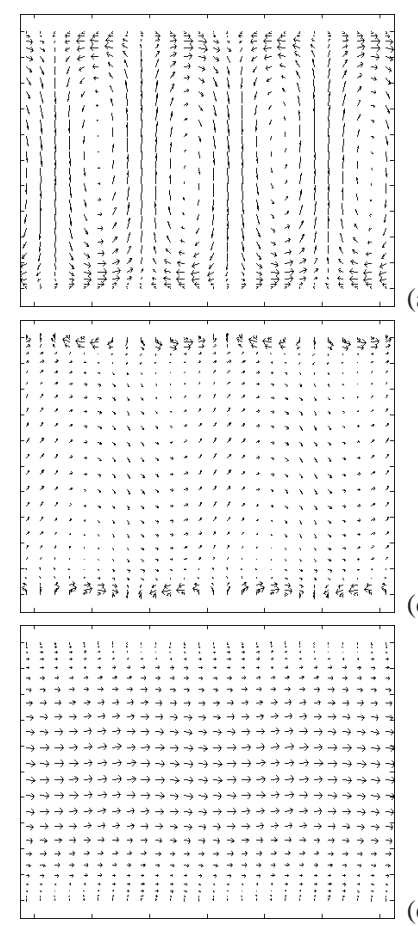

(c)
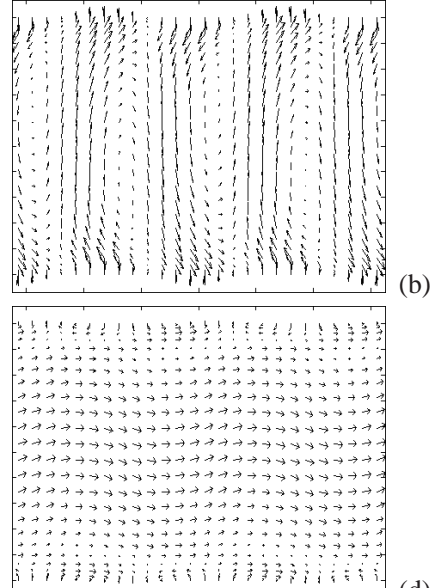

(d)

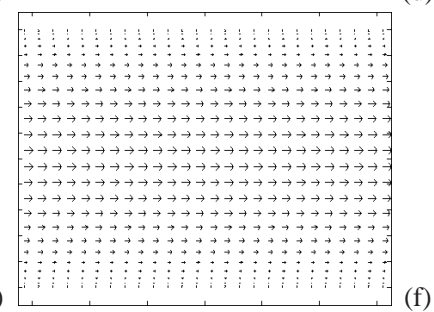

Fig. 4. Velocity field vs time. (a) due to an initial perturbation the flow becomes turbulent; (b) first iteration of the control law; (c) and (d) intermediate state of the controlled flow; (e) the flow becomes laminar; (f) the control law converged, the flow is laminar in an unstable state.

\section{Simulation RESUlts}

The simulation results are based on the code provided in [21]. As in [5], [8], [6], we consider $R_{e}=10000$ and a channel length $L_{c}=4 \pi$ (see Fig. 1). The scalar $r$ involved in (18) has been set to 2.5 while $\lambda$, involved in the desired behavior of the drag, has been set to 0.5 . The dimension of the state vector $M$ has been set to 40 .

First, we present on Fig. 4 the behavior of the velocity field during the servoing. The flow is first not controlled and becomes thus turbulent due to the presence of a temporal perturbation and because its unstable state. After the application of the input signal, the flow tends towards a laminar behavior but, of course, still in an unstable state.

We compare our approach with the most relevant ones, that is [5], [17], [19]; [15], [16] are similar in mind than [17]. We respectively denote these approaches $P, L Q R_{2}$ and $L Q R_{1}$, we denote ours by $P V S$. Let us briefly describe these works. In [5], a proportional output feedback control is used, the output is the wall shear stress. Therefore, vanishing the shear stress also vanishes the drag. In this approach the kinetic energy density is not considered. In [19] the kinetic energy density is minimized by using a LQR approach. The matrix $\mathrm{Q}$ involved in (10) is used in the cost function to minimize. Note that is an indirect way to minimize the drag since the drag vanishes when the kinetic energy density does. Finally, in [17], the wall shear stress is minimized also through a LQR approach.

Note that we cannot exactly compared our approach with these works since none of them simultaneously minimizes the drag and the kinetic energy density. The results are
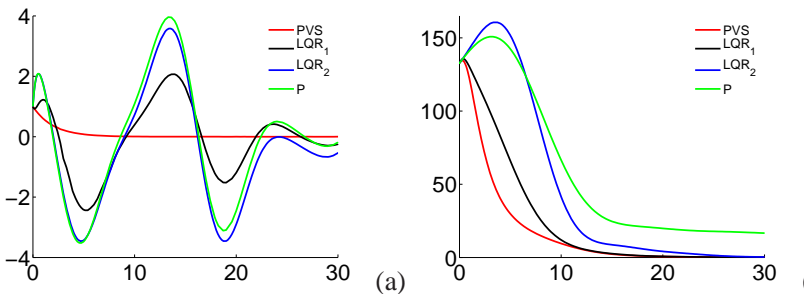

(b)

Fig. 5. Comparison of the different approaches ( $x$ axis in second). (a) drag vs time; (b) kinetic energy density vs time.

depicted on Fig. 5. More precisely, Fig. 5a depicts the behavior of the drag versus time while Fig. 5b depicts the behavior of the kinetic energy density versus time. As expected, our approach provides better results than the other approaches. A nice decreasing of the drag and the kinetic energy density are observed. The $L Q R_{1}$ approach leads also to a nice minimization of the kinetic energy density but the (indirect) minimization of the drag is not satisfactory. Both methods based on the minimization of the shear stress $(P$ and $L Q R_{2}$ ) lead to a worse behavior than $P V S$ and $L Q R_{1}$. Note also that, except for our approach, the decreasing of the drag towards zero is very low.

However, the input signal provides both by the $P$ and $L Q R_{2}$ methods are very low in contrast to the $P V S$ and $L Q R_{1}$ approaches (see Fig. 6). Therefore, by tuning the matrix $\mathbf{R}$ involved in the $L Q R_{2}$ approach or the gain for the $P$ approach better results could be expected. Unfortunately, it is not the case as proved by the results depicted on Fig. 7. In particular, oscillations occur in the kinetic energy density (Fig. 7b).

Fig. 8 describes the variation of the kinetic energy density for various choices of $\lambda$, as can be seen this kinetic energy density does not depend, in practice, on this value. That means that the way the drag is reduced does not influence the way the kinetic energy density is reduced.

The next simulation concerns the behavior of the system when noisy measurement are considered. The results are depicted on Fig. 9, with or without noise. Respectively the drag, the kinetic energy density and the norm of the input signal are represented on Fig. 9a, Fig. 9b and Fig. 9c. A Gaussian noise with a standard deviation of 0.3 has been added to the velocity fields. The number of pixels $N_{x}$ has been set to 2048. As can be seen, despite this very low value, very good results are obtained. In particular, the convergence of the control law is ensured, as well as the decrease of the drag. Note that, contrary to our vision-based estimation scheme, all the works previously described are very sensitive to measurement noise. The control law may be diverged (see [1] for more details).

\section{CONClusion}

We have shown in this paper that partitioned visual servo control is an efficient way to simultaneously minimize the drag and the kinetic energy density contrary to the existing methods. Moreover, we have shown that in practice, the way the drag is reduced does not influence the way the kinetic energy density is reduced. In addition, we have proved that 


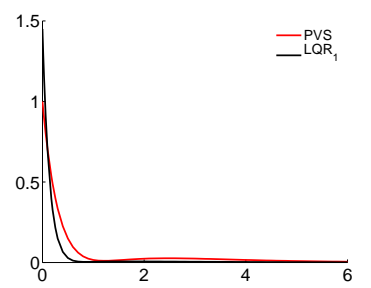

(a)

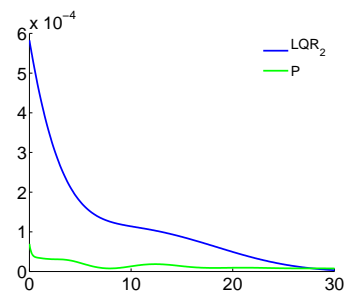

(b)

Fig. 6. Comparison of the different approaches ( $x$ axis in second). Norm of the input signal $\mathbf{u}(t)$ vs time.
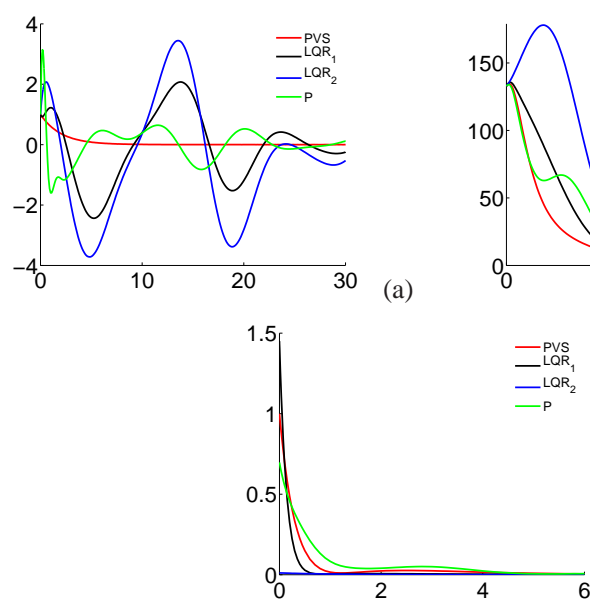

(c)

Fig. 7. Comparison of the different approaches ( $x$ axis in second). (a) drag vs time; (b) kinetic energy density vs time (c) Norm of the input signal $\mathbf{u}(t)$ vs time.

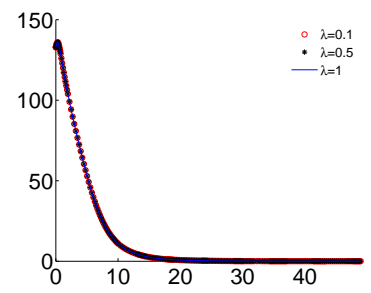

Fig. 8. Behavior of the kinetic energy density for different values of $\lambda(x$ axis in second).

our approach is very robust against measurement noise. This nice propriety is due to the large amount of flow velocities available through the computation of the optical flow. In contrast, the existing methods are sensitive to noise since the state estimation is based on shear stress measurements on only few points.

\section{REFERENCES}

[1] R. Tatsambon Fomena and C. Collewet, "Fluid flow control: a visionbased approach," International Journal of Flow Control, 2012, To appear.

[2] P. Corke and S. Hutchinson, "A new partitioned approach to imagebased visual servo control," IEEE Trans on Robotics and Automation, vol. 17, no. 4, pp. 507-515, August 2001.

[3] F. Chaumette and S. Hutchinson, "Visual servo control, Part II: Advanced approaches," IEEE Robotics and Automation Magazine, vol. 14, no. 1, pp. 109-118, March 2007.

[4] A. Flaig, "Eco-efficient by design," in Challenges for aerodynamics engineers for future aircraft design, 8th World Congress on Computational Mechanics, Venice, Italy, 2008.

[5] S. Joshi, J. Speyer, and J. Kim, "A system theory approach to the feedback stabilization of infinitesimal and finite amplitude disturbances in plane Poiseuille flow," Journal of Fluid Mechanics, vol. 332, pp. 157184, 1997.

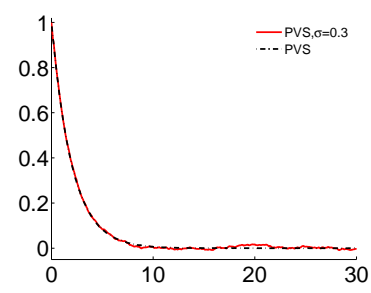

(a)

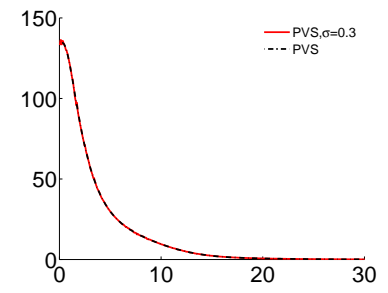

(b)

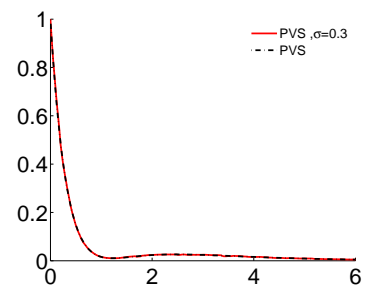

(c)

Fig. 9. Behavior when measurement noise is considered ( $x$ axis in second) (a) drag vs time; (b) kinetic energy density vs time; (c) Norm of the input signal $\mathbf{u}(t)$ vs time.

[6] T. Bewley and S. Liu, "Optimal and robust control and estimation of linear paths to transition," Journal of Fluid Mechanics, vol. 365, pp. 305-349, 1998.

[7] B. Protas and J. Wesfreid, "Drag force in the open-loop control of the cylinder wake in the laminar regime," Physics of Fluids, vol. 14, no. 2, pp. 810-826, February 2002.

[8] J. McKernan, G. Papadakis, and J. Whidborne, "A linear state-space representation of plane poiseuille flow for control design- a tutorial," International Journal of Modelling, Identification and Control, vol. 1, no. 4, pp. 272-280, 2006.

[9] D. Heitz, E. Mémin, and C. Schnörr, "Variational fluid flow measurements from image sequences: synopsis and perspectives," Exp. Fluids, vol. 48, no. 3, pp. 369-393, 2010.

[10] F. Chaumette and S. Hutchinson, "Visual servo control, Part I: Basic approaches," IEEE Robotics and Automation Magazine, vol. 13, no. 4, pp. 82-90, December 2006.

[11] B. Espiau, F. Chaumette, and P. Rives, "A new approach to visual servoing in robotics," IEEE Trans. on Robotics and Automation, vol. 8, no. 3, pp. 313-326, June 1992.

[12] F. Bonin-Font, A. Ortiz, and G. Oliver, "Visual navigation for mobile robots: a survey," Journal of Intelligent \& Robotic Systems, vol. 53, no. 3, pp. 263-296, 2008.

[13] T. Bewley, H. Choi, T. Temam, and P. Moin, "Optimal feedback control of turbulent channel flow," 1993, annual research briefs.

[14] J. Kim, "Control of turbulent boundary layers," Physics of Fluids, vol. 15, no. 5, pp. 1093-1105, May 2003.

[15] S. Joshi, J. Speyer, and J. Kim, "Finite dimensional optimal control of poiseuille flow," Journal of Guidance, Control, and Dynamics, vol. 22, no. 2, pp. 340-348, 1999.

[16] K. H. Lee, L. Cortelezzi, J. Kim, and J. L. Speyer, "Application of robust reduced-order controller to turbulent flows for drag reduction," Physics of Fluids, vol. 13, no. 5, pp. 1321-1330, 2001.

[17] L. Cortelezzi, K. Lee, J. Kim, and J. Speyer, "Skin-friction drag reduction via robust reduced-order linear feedback control," International Journal of Computational Fluid Dynamics, vol. 11, pp. 79-92, 1998.

[18] J. F. Whidborne and J. McKernan, "On the minimization of maximum transient energy growth," IEEE Trans. on Automatic Control, vol. 52, no. 9, pp. 1762-1767, September 2007.

[19] J. McKernan, J. Whidborne, and G. Papadakis, "Linear quadratic control of plane poiseuille flow - the transient behaviour," International Journal of Control, vol. 80, no. 12, pp. 1912-1930, December 2007.

[20] O. Aamo, M. Krstic, and T. Bewley, "Control of mixing by boundary feedback in 2d channel flow," Automatica, no. 39, pp. 1597-1606, 2003.

[21] J. McKernan, "Control of plane Poiseuille flow: a theoretical and computational investigation," Ph.D. dissertation, Cranfield University, 2006.

[22] L. Cattafesta and M. Sheplak, "Actuators for active flow control," Annual Review of Fluid Mechanics, vol. 43, no. 1, pp. 247-272, 2011.

[23] S. Orszag, "Accurate solution of the Orr-Sommerfeld stability equation," Journal of Fluid Mechanics, vol. 50, no. 4, pp. 689-703, 1971. 